\title{
Temperature-Regulated Regiodivergent Synthesis via Alkene Migratory Hydroalkylation
}

\author{
Jia-Wang Wang ${ }^{1,2}$, De-Guang Liu' ${ }^{1,2}$, Zhe Chang ${ }^{1,2}$, Zhen $\mathrm{Li}^{1}$, Yao Fu' ${ }^{1}$ Xi Lu ${ }^{1, *}$
}

${ }^{1}$ School of Chemistry and Materials Science, CAS Key Laboratory of Urban Pollutant Conversion, Anhui Province Key Laboratory of Biomass Clean Energy, University of Science and Technology of China, Hefei 230026, China ${ }^{2}$ These authors contributed equally: Jia-Wang Wang, De-Guang Liu, Zhe Chang.

*E-mail: luxi@mail.ustc.edu.cn.

\begin{abstract}
An approach for reliable and predictable carbon-carbon or carbon-heteroatom bond formation that produces either regioisomer starting from the same raw materials, also known as a regiodivergent methodology, is highly desirable. Altering the chemical reaction variables, including catalysts, ligands, solvents, or other additives, were the predominant strategy for the implementation of regiodivergent features in metal-catalysed organic synthesis. The achievement of switchable selectivity using quickly and conveniently controlled physical variables constitutes a desirable goal with an intriguing method of attainment. Herein, we report our discovery that temperature-regulated switchable site-selectivity can be achieved in alkene (migratory) functionalization. Judicious selection of reaction temperatures, one of the most easily changed variables, led to protocols that provide the regiodivergent alkylation products starting from a single alkene substrate. This protocol allows for the convenient synthesis of $\alpha$ - and $\beta$-branched protected amines, both of which are substantially important to the pharmaceutical chemistry and biochemistry fields. In addition, enantioenriched $\beta$-branched alkyl amines could be accessed in a catalytic asymmetric variant manner. This work may inspire more research interests in metal-catalysed regiodivergent reaction discovery using easily changed physical variables.
\end{abstract}

\section{Introduction}

Reliable and predictable catalytic reactions that produce either regioisomer when starting from the same raw materials, also called regiodivergent reaction methodologies, are highly desirable. The different regioisomers can be prepared by properly interchanging the catalysts or altering the reaction conditions. ${ }^{1,2}$ Therefore, these regiodivergent reactions exhibit higher productivity, better atom- and step-economy, and better cost-efficiency and are quickly gaining favour in synthetic organic chemistry. As was summarized by Nájera and coworkers, different catalysts, ligands, solvents, or additives, were the predominant strategy for the implementation of 
regiodivergent features in metal-catalysed organic synthesis. ${ }^{1}$ The achievement of switchable selectivity using quickly and conveniently controlled physical variables constitutes a desirable goal with an intriguing method of attainment. Reaction temperature, one of the most easily changed physical variables in organic synthesis, can affect the regioselectivity of reactions (Figure 1a). For example, the Kolbe-Schmitt reaction, the named reaction for the synthesis of salicylic acid, is a thermodynamically controlled equilibrium reaction. A lower reaction temperature is conducive to the formation of ortho-isomers, and a higher reaction temperature is conducive to the formation of para-isomers. Fries rearrangement reaction also exhibits a temperature-regulated regiodivergence. In another example, Martin and coworkers reported the discovery of a temperature-dependent regiodivergent remote carboxylation of alkyl halides with carbon dioxide. ${ }^{3}$

The site-specific formation of carbon-carbon or carbon-heteroatom bonds provides new insight to chemists during retrosynthetic analysis. ${ }^{4-6}$ Alkene (migratory) functionalization represents an attractive method for the construction of carbon-carbon bonds and carbon-heteroatom bonds. ${ }^{7-12}$ The in situ generation of alkyl-metallic intermediates from widely accessible alkenes circumvents the time-consuming use of reactive and often sensitive organometallic reagents in traditional electrophile-nucleophile cross-coupling, which improves our everexpanding synthetic toolkit for forming $s p^{3}$ hybridized linkages. ${ }^{13-17}$ In addition, carbon-carbon bond formation on remote and unfunctionalized sites enabled by the chain-walking process, a formal translocation of the original active site to a nonspecific position along with the carbon skeletons, allows for the synthesis of complex structures that would otherwise be difficult to prepare. ${ }^{18-25}$ In the context of alkene functionalization, both catalysts and ligands could regulate the regiodivergence of hydrofunctionalization ${ }^{26-29}$ and difunctionalization process $^{30-34}$. In consideration of the essential problem that to achieve switchable selectivity using easily changed and controlled physical variables, switching reaction temperatures to provide the regiodivergent products starting from a single alkene substrate would be an ideal choice.

Inspired by these achievements, we sought to explore the influence of temperature on accurate and switchable site selectivity in alkene functionalization reactions. Herein, we report our discovery that switchable selectivity can be achieved in nickel-hydride-catalysed alkene migratory hydroalkylation (Figure 1c). Judicious selection of reaction temperatures led to protocols that provide the regiodivergent $\alpha$ - and $\beta-\mathrm{C}-\mathrm{H}$ alkylation products and start from a single alkene substrate. This reaction also exhibits regioconvergence, delivering the predictable regioselective product with high regioselectivity from the arbitrary isomer or even isomeric mixtures of the alkene substrates. This protocol allows for the convenient synthesis of $\alpha$ - and $\beta$-branched alkyl amines, each of which are substantially important to the pharmaceutical chemistry and biochemistry fields. Furthermore, enantioenriched $\beta$-branched alkyl amines could be accessed in a catalytic asymmetric variant manner, which 
provides a convenient synthetic route to enantioenriched $\beta$-branched alkyl amines that are substantially important to the pharmaceutical chemistry and biochemistry fields (Figure 1d). ${ }^{35}$

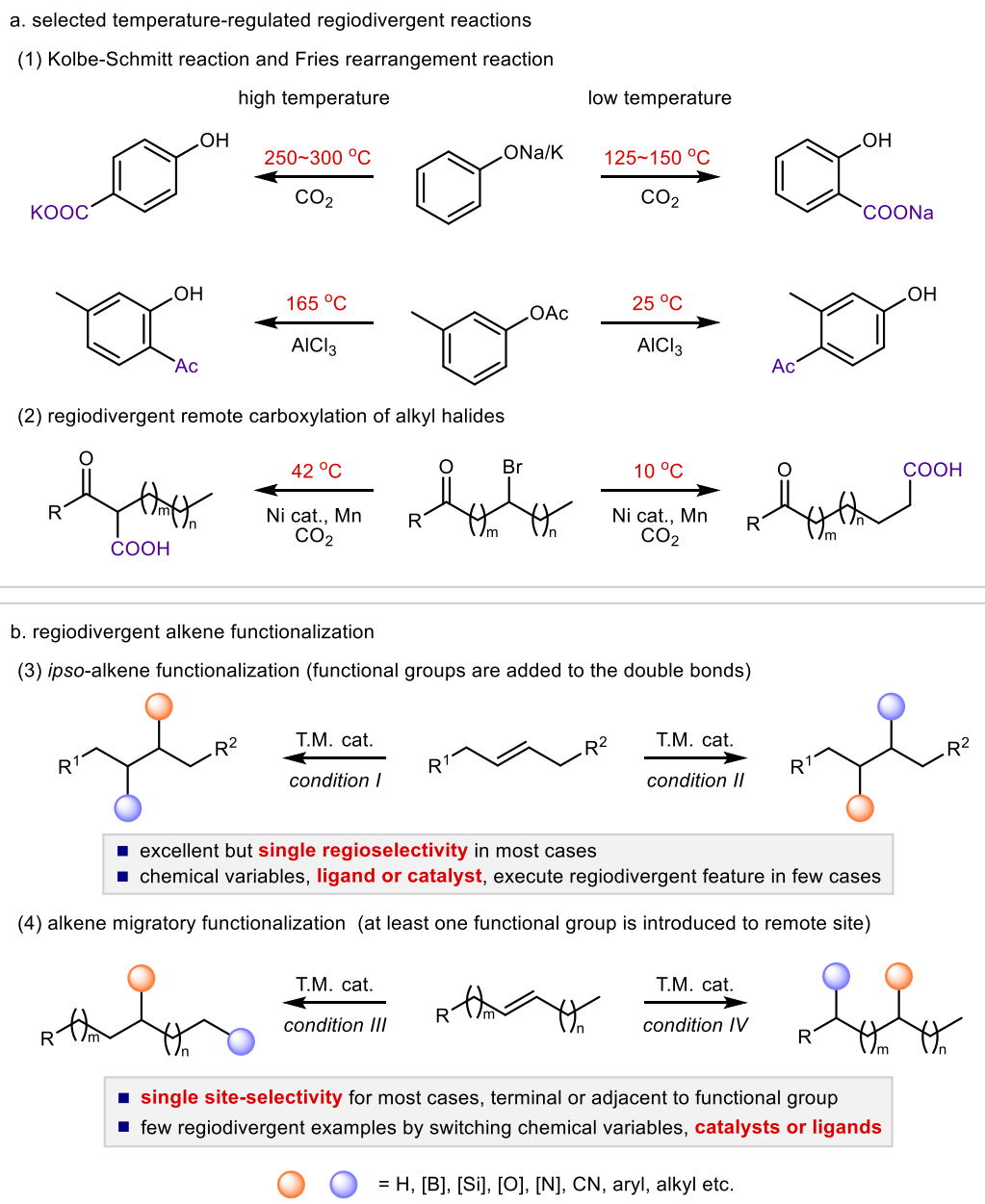

c. this work: regiodivergent alkene migratory hydyroalkylation

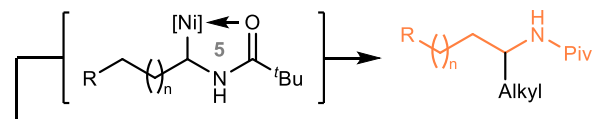
$\mathrm{P}_{\mathrm{n}}^{\mathrm{N}}{ }_{\text {Piv }}^{\mathrm{H}}$

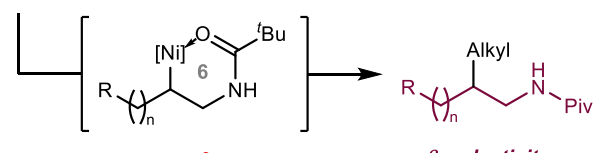

$10^{\circ} \mathrm{C}$

$\beta$ - selectivity

- temperature-regulated regiodivergent selectivity, maneuverable

- regioconvergent remote $\mathrm{C}\left(s p^{3}\right)$-H alkylation, value-added process

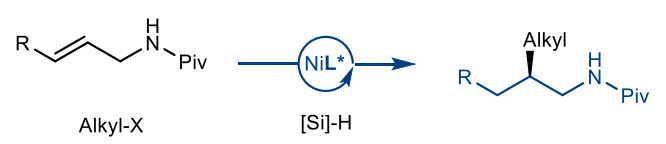

- asymmetric variant for the synthesis of $\beta$-branched chiral amines

d. examples of physiologically active $\beta$-branched chiral amines
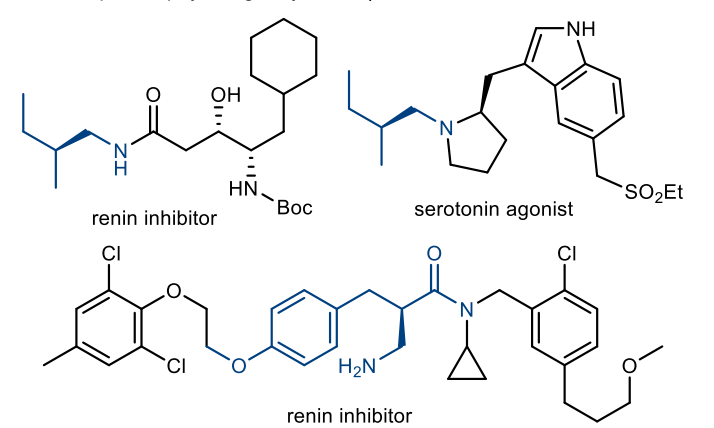

Figure 1. Regiodivergent alkene functionalization and our stratergy: temperature-regulated regiodivergent and regioconvergent alkene migratory hydroalkylation. T.M., transition-metal; Ac, acetyl; Piv, pivaloyl; Boc, $t$ butoxycarbonyl.

\section{Results}

Reaction discovery and optimization. The widespread nature of $C\left(s p^{3}\right)-C\left(s p^{3}\right)$ structures makes it essential for organic synthetic chemists to improve the construction of these common bonds. ${ }^{36}$ The developments in pharmaceutical chemistry also create a need for new synthetic methods that facilitate alkyl carbon centres. ${ }^{4-6}$ The utilization of regiodivergent alkene hydroalkylation to access the $\mathrm{C}\left(s p^{3}\right)-\mathrm{C}\left(s p^{3}\right)$ bond was highly desired. Consistent with our continuous interest in alkene hydroalkylation, ${ }^{14,16,37-39}$ we commenced our study by exploring the migratory $\beta$-selective hydroalkylation of 1a with alkyl iodide 2 a to access protected $\beta$-branched alkyl amines. We systematically screened all reaction parameters and determined that a combination of $\mathrm{NiBr}_{2}$ (diglyme) and bisoxazoline ligand $\mathbf{L}$ with $(\mathrm{EtO})_{3} \mathrm{SiH}$ and $\mathrm{K}_{3} \mathrm{PO}_{4}\left(\mathrm{H}_{2} \mathrm{O}\right)$ in DMAc at $10{ }^{\circ} \mathrm{C}$ was critical for success, and an $86 \% \mathrm{GC}$ 
yield and a 12:1 r.r. were obtained. More detailed information on the changes of each reaction parameter is summarized in Figure 2a. Careful selection of the bisoxazoline ligand played an extremely vital role in the success of this reaction. The structurally related bipyridine ( $\mathbf{L} 1$ and $\mathbf{L} 2)$, bioxazoline $\left(\mathbf{L}_{3}\right)$, and pyridine-oxazoline $\left(\mathbf{L}_{\mathbf{4}}\right)$ ligands were tested and gave moderate to good yields with poor regioselectivities (1:3.6 1.7:1 r.r.). A small amount of the target product was observed when we used a diamine ligand ( $\mathbf{L}_{\mathbf{5}}$ ). Many other nickel catalysts could be used, such as $\mathrm{NiBr}_{2}$ and $\mathrm{NiI}_{2}$ (entry 2), but $\mathrm{Ni}\left(\right.$ acac) ${ }_{2}$ was invalid for this reaction (entry 3). Many other oxygenbearing silanes, such as DEMS, PMHS and $(\mathrm{MeO})_{3} \mathrm{SiH}$, could be used instead of (EtO) $)_{3} \mathrm{SiH}$ with slightly decreased coupling efficiency and comparable regioselectivity (entry 4). $\mathrm{Ph}_{2} \mathrm{SiH}_{2}$ delivered a gratifying 8.1:1 regioisomeric ratio with a low $22 \%$ total yield (entry 5 ), while the less electrophilic hydrosilane $\mathrm{Et}_{3} \mathrm{SiH}_{\mathrm{H}}$ provided no cross-coupling product (entry 6). $\mathrm{K}_{3} \mathrm{PO}_{4}$ without crystal water also performed well (entry 7 ); $\mathrm{Na}_{2} \mathrm{CO}_{3}$ provided excellent regioselectivity, but the coupling yield was unsatisfactory (entry 8); lower yields and poor regioselectivities were obtained using a medium strength base such as KF or CsF (entry 9). Various solvents were compared to the optimal solvent DMAc. Amide solvent, DMF, afforded a high regioisomeric ratio with a slightly decreased $75 \%$ yield (entry 10 ). Polar aprotic $\mathrm{CH}_{3} \mathrm{CN}$, ether THF, and hydrocarbon solvents such as toluene and DCE were inferior and exhibited significantly reduced regioselectivities (entry 11). Finally, this hydroalkylation reaction could not be performed without nickel catalysts (entry 12 ); a halved yield of $42 \%$ with a roughly equal 11:1 regioisomeric ratio was obtained in the absence of the bisoxazoline ligand $\mathbf{L}$ (entry 13 ).

With respect to migratory $\alpha$-selective hydroalkylation, we found that the increased reaction temperature led to a reversed $\alpha$-selectivity that allowed for the production of 4 aa with both high regioselectivity and an excellent coupling yield (Figure 2b). A good GC yield of $97 \%$ and a $94 \%$ isolated yield with a superb regioselectivity profile (>20:1 r.r.) resulted under similar reaction conditions for the migratory $\beta$-selective hydroalkylation shown in Figure 2a, and only the modification was the increase in reaction temperature to $100{ }^{\circ} \mathrm{C}$. Therefore, judicious selection of reaction temperatures, one of the most easily changed variables in organic synthesis, led to protocols that provide the regiodivergent $\alpha$ - and $\beta-\mathrm{C}-\mathrm{H}$ alkylation products starting from a single alkene substrate.

Finally, we determined that enantioselective $\beta$-selective hydroalkylation could be realized (Figure 2c). With slight modifications to the solvent and reaction temperature based on the reaction conditions for migratory $\beta$ selective hydroalkylation, the protected allylamine 3 a and alkyl iodide $\mathbf{2 a}$ coupled well to deliver the enantioenriched $\beta$-branched amine 6aa with a 96\% GC yield, an 89\% isolated yield and a good regio- and enantioselectivity profile (9.2:1 r.r. and 94\% e.e.). The mixed $\mathrm{DMF} /{ }^{\mathrm{t}} \mathrm{BuOH}$ solvent exhibited a slight advantage over the single solvents, such as DMAc, DMF, or THF, with regard to both regio- and enantioselectivity (entries 2 4). MeOH was inferior because its use resulted in low alkene conversion (entry 5). A slightly increased reaction 
temperature at $21{ }^{\circ} \mathrm{C}$ rather than $10{ }^{\circ} \mathrm{C}$ improved the yield (entry 6). Comparative results on other bisoxazoline ligands are also listed (see Supplementary Table 3 for more details). More benzyl substitutions on the linkage of bisoxazoline improved the yield and enantioselectivity ( $\mathbf{L}^{*}$ versus $\mathbf{L 6}$ versus $\mathbf{L}_{7}$ ). Aryl substitution on the oxazoline ring performed better than other substitutions, even structurally similar indan substituents $\left(\mathbf{L}^{*}\right.$ versus L8).

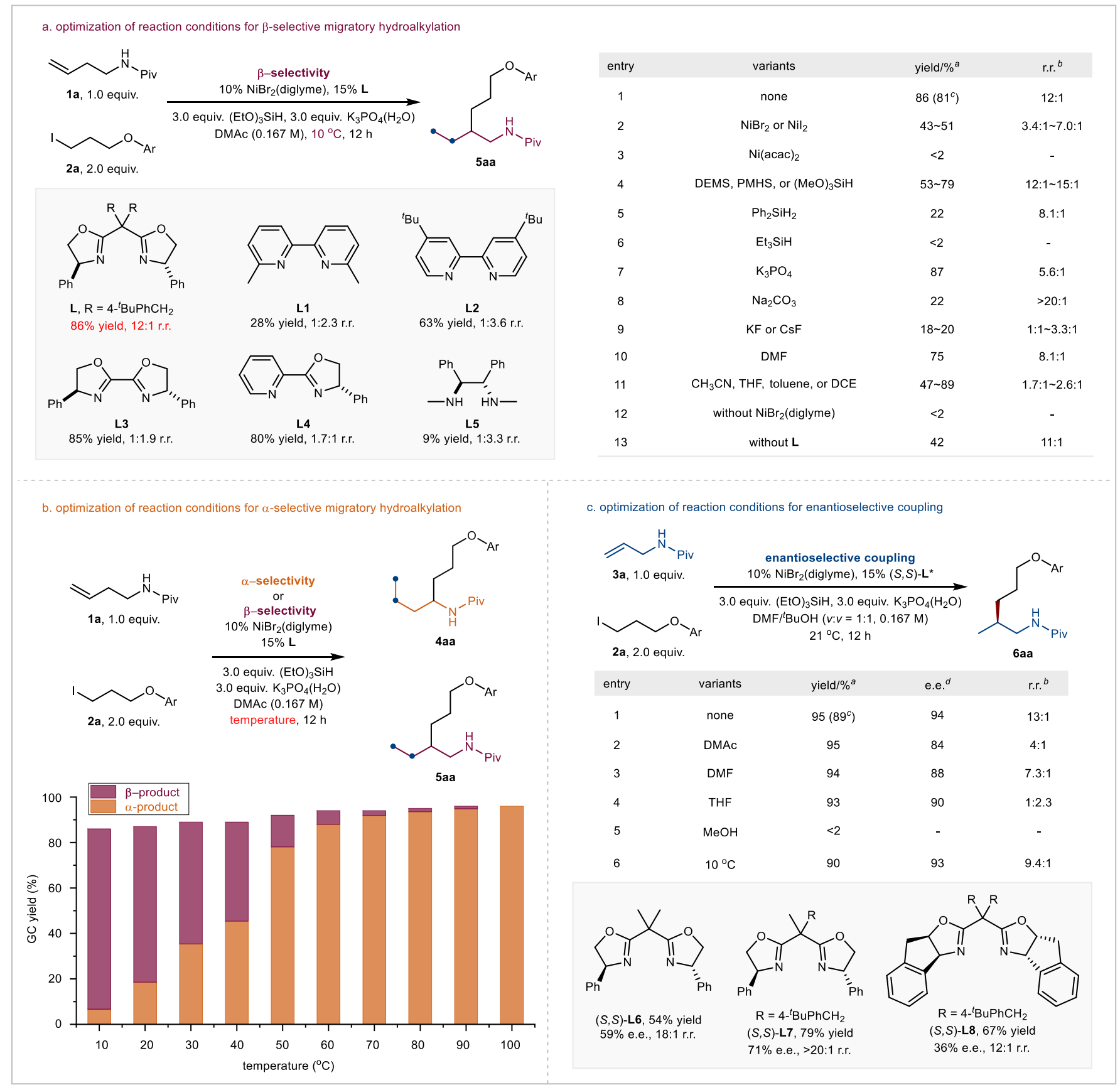

Figure 2. Optimization of reaction conditions. Reactions were carried out under an argon atmosphere. Conditions: 1 a (o.10 mmol, 1.0 equiv), 2 a (o.20 mmol, 2.0 equiv), nickel catalyst (o.01 mmol, $10 \mathrm{~mol} \%)$, L (o.015 mmol, 15 mol\%), silane (o.30 mmol, 3.0 equiv), base (o.30 mmol, 3.o equiv), solvent (o.6o mL, o.167 M), $12 \mathrm{~h}$. ${ }^{a}$ Yield refers to the total yield for the mixture of all regioisomers. ${ }^{b}$ r.r. refers to the regioisomeric ratio of the major product to the sum of all other isomers. For alkene migratory hydroalkylation and enantioselective 
hydroalkylation, $\alpha$-, $\beta$ - and linear-selective products were observed; other regioisomers could hardly be detected. Yields and regioisomeric ratios were determined by GC analysis with triphenylmethane as an internal standard. ${ }^{c}$ Isolated yield in parentheses. ${ }^{d}$ The e.e. value was determined by high-performance liquid chromatography (HPLC). Ar, 2-naphthyl; acac, acetylacetanoate; DEMS, diethoxymethylsilane; PMHS, polymethylhydrosiloxane; DMAc, N,N-dimethylacetamide; DMF, N,N-dimethylformamide; THF, tetrahydrofuran; DCE, 1,2dichloroethane.

Substrate scope of migratory hydroalkylation. We sought to examine the generality of this migratory hydroalkylation reaction with suitable reaction conditions by exploring a wide range of alkenes and alkyl iodides (Figure 3). An array of alkene substrates with different steric hindrances and chain lengths smoothly underwent alkene isomerization hydroalkylation, convincingly demonstrating the reliability of temperature-regulated regioselectivity. The regioselectivity was precisely controlled by the reaction temperature: high temperature $\left(100{ }^{\circ} \mathrm{C}\right)$ was always $\alpha$-selective, whereas low temperature $\left(10^{\circ} \mathrm{C}\right)$ was $\beta$-selective. In addition, a complete lack of isomerization of the alkyl halides was observed. Both terminal aliphatic alkenes (4aa and 5aa, 4ca and $5 \mathbf{c a}$ ) and internal alkenes (4ba and $\mathbf{5} \mathbf{b a}$ ) with different chain lengths performed well. Alkane substrates $\mathbf{1 d}$ and $\mathbf{1 e}$ have potentially competitive benzylic or $\alpha$-ester active reaction sites. These substrates delivered prospective $\alpha$ - or $\beta$ selective migratory hydroalkylation products (4da and $5 \mathbf{d a}, \mathbf{4}$ ea and 5ea, respectively), indicating that the amide group displays a higher priority directing ability over an aromatic ring or an ester group. However, $\alpha$-selective migratory hydroalkylation exhibits better regiocontrol capability and applies to a broader range of alkene substrates than $\beta$-selective migratory hydroalkylation. Both method A and method B were performed on the 1,1disubstituted alkene If. $\alpha$-Branched product 4 fa was obtained smoothly under method A conditions, while method B produced the unexpected linear product $\mathbf{5} \mathbf{f a}$ (see Supplementary Figure 26 for DFT calculation results). A wide range of acyl groups other than pivaloyl (Piv, 4aa and 5aa) could be used as the amine protecting group, and the directing groups include acetyl (Ac, $\mathbf{4 g a}$ ) and benzoyl (Bz, 4 ha and $\mathbf{5 h a}$ ). In addition, tertiary amides in the absence of the N-H bond were also suitable as auxiliary directing groups. For example, 2-pyrrolidinone (4ia and 5ia), 3-morpholinone (4ja and 5ja), phthalimide (4ka), and 2-oxazolidone (5la) were successfully used as protecting groups and directing groups. tert-Butyloxycarbonyl (Boc), a ubiquitous amine protecting group resulted in high $\alpha$-selective migratory hydroalkylation (5ma).

A variety of alkyl iodides with various functional groups were readily converted to the target products in moderate to good yields (52 91\%) and with high regioisomeric ratios (5.2:1 >20:1 r.r.). We successfully transformed the alkyl iodides $\mathbf{2 b}$ and $\mathbf{2 c}$ to a series of products (4ab and $5 \mathbf{a b}, 4 \mathbf{a c}$ and $5 \mathbf{a c}$ ), which indicates that the regiodivergence of the reaction does not rely on alkyl halide substrates bearing specific heteroatoms. Many 
valuable synthetic functional groups including esters (4ad and 5ad), trifluoromethyls (4ae and $5 \mathbf{a e}$ ), and various heterocyclic groups such as thiophene (4af and $5 \mathbf{a f}$ ) and furan (4ag and $5 \mathbf{a g}$ ) were compatible with the use of mild and robust reaction conditions. This reaction is highly chemoselective to electrophilic aryl chloride (4ah and $\mathbf{5} \mathbf{a h}$ ), aryl tosylate (4ai and $\mathbf{5} \mathbf{a i}$ ), and nucleophilic arylboronate (4aj and $\mathbf{5} \mathbf{a j}$ ), thus allowing further transformation through other cross-coupling reactions.

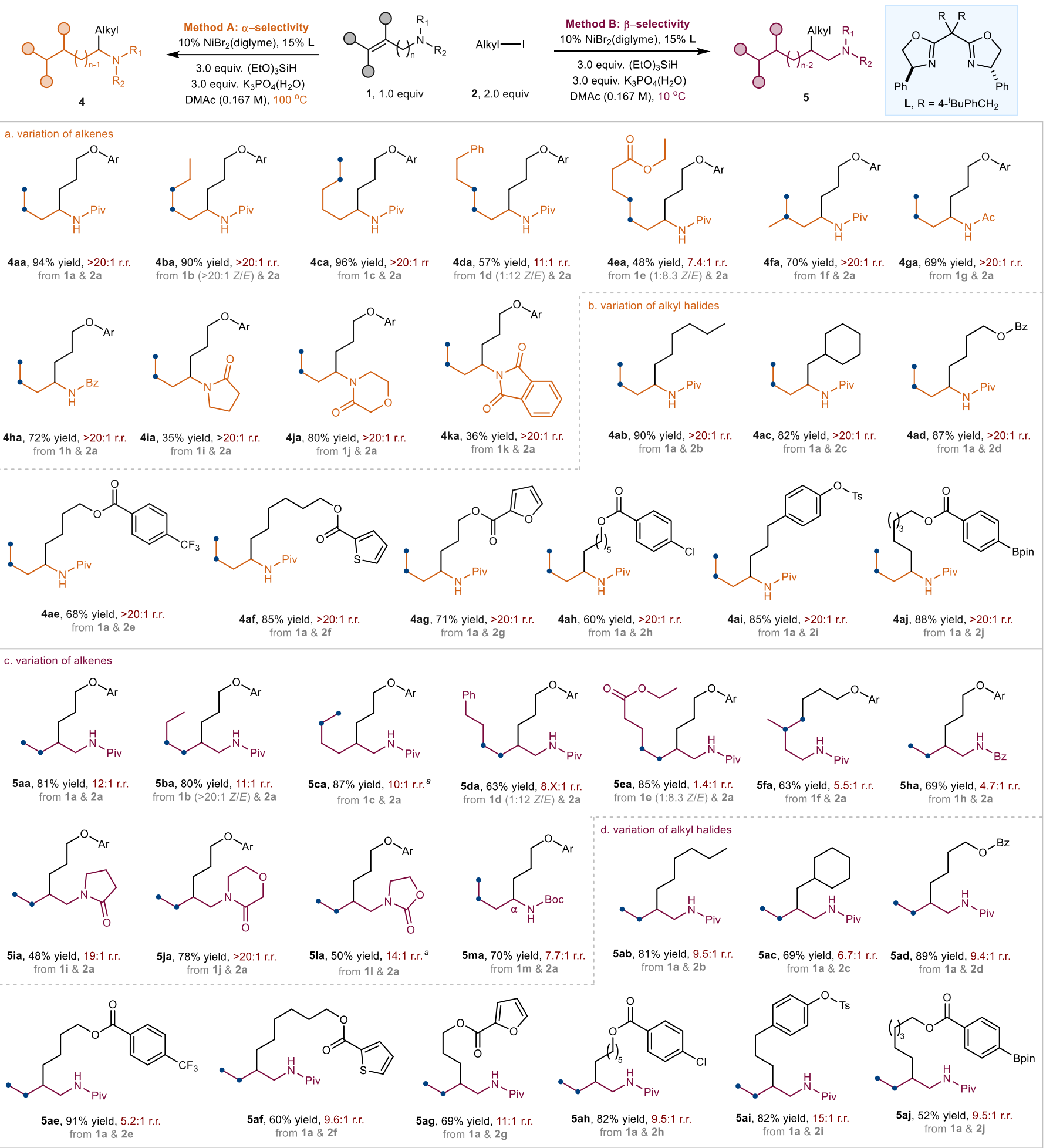

Figure 3. Temperature-regulated migratory hydroalkylation. Method A: alkene (o.20 mmol, 1.o equiv), alkyl 
halide (o.40 mmol, 2.0 equiv), $\mathrm{NiBr}_{2}$ (diglyme) (o.02 mmol, $10 \mathrm{~mol} \%$ ), L (o.030 mmol, $15 \mathrm{~mol} \%$ ), (EtO) 3 SiH (o.6o mmol, 3.o equiv), $\mathrm{K}_{3} \mathrm{PO}_{4}\left(\mathrm{H}_{2} \mathrm{O}\right)$ (o.6o mmol, 3.0 equiv), DMAc $(1.2 \mathrm{~mL}, 0.167 \mathrm{M}), 100{ }^{\circ} \mathrm{C}, 12 \mathrm{~h}$, isolated yield. The r.r. value was determined by GC or ${ }^{1} \mathrm{H}$ NMR analysis. Method B is similar to Method A, except for the reaction temperature. For Method B, the reaction mixture was stirred at $10{ }^{\circ} \mathrm{C}$ for $12 \mathrm{~h} .{ }^{a}-5{ }^{\circ} \mathrm{C}$. Bz, benzyl; Ts, tosyl; Bpin, boronic acid pinacol ester.

Scope of enantioselective $\boldsymbol{\beta}$-selective hydroalkylation. The scoping study results for the production of enantioenriched $\beta$-branched alkyl amines through enantioselective $\beta$-selective hydroalkylation are summarized in Figure 4. The reaction was conducted smoothly with various alkyl bromides and iodides and protected allylamines and resulted in the production of the target products in good to excellent yields (45 96\%) with high regioisomeric ratios and enantioinduction. Compared to the model reaction with $\mathbf{2 a}$, both the selected alkyl iodides (2k and $\mathbf{2} \mathbf{l})$ that lacked any heteroatoms and the substrates containing sulfur or phosphonate atoms (2r and 2s) were successfully transformed to their corresponding products (6ak, 6al, 6ar, 6as) without significant differences in coupling efficiency or enantioselectivity. This asymmetric hydroalkylation tolerated a wide range of functional groups, including ester (6ad), trifluoromethyl (6am), trifluoromethoxy (6an), cyano (6ao), and aryl chloride (6ah). Heterocycles such as phthalimide (6ap), furan (6ag), and thiophene (6aq) remained unchanged in this hydroalkylation reaction. Although the reaction conditions involve a medium-strength base $\mathrm{K}_{3} \mathrm{PO}_{4}\left(\mathrm{H}_{2} \mathrm{O}\right)$, compounds with keto carbonyl groups (6at, 6au) were well tolerated and thus demonstrated the excellent compatibility of this reaction with base-sensitive functional groups. Many internal alkenes were proven to be suitable substrates. Excellent enantioselectivities (88 96\% e.e.) were obtained in all cases. In general, alkenes with less bulky substituents provided good yields (6ba, 6da), while bulkier substrates resulted in moderate coupling efficiency $(\mathbf{6 c J})$. Notably, the $Z / E$ configuration of the alkene indeed affects the regio- and enantioselectivity (6da). Another limitation of this reaction is that the regioselectivity of the reaction is not satisfying when utilizing substrates with intolerable ester groups in the alkyl chain (6ea, 6fa), which suggests that there is a need to further optimize the reaction conditions. Cinnamylamine derivatives have potentially competitive benzylic active reaction sites. We examined several cinnamylamine derivatives with different electronic properties of the aryl ring. Both electroneutral (6gb), electron-rich (6hb, 6ia), and electron-deficient (6ja, 6ka) compounds gave uniformly good coupling yields and regio- and enantioselectivities.

Finally, the absolute configuration of 6aJ was determined by comparison with that of the standard product (see Supplementary Note 2 for more details). We assigned the corresponding absolute configuration to other products. 

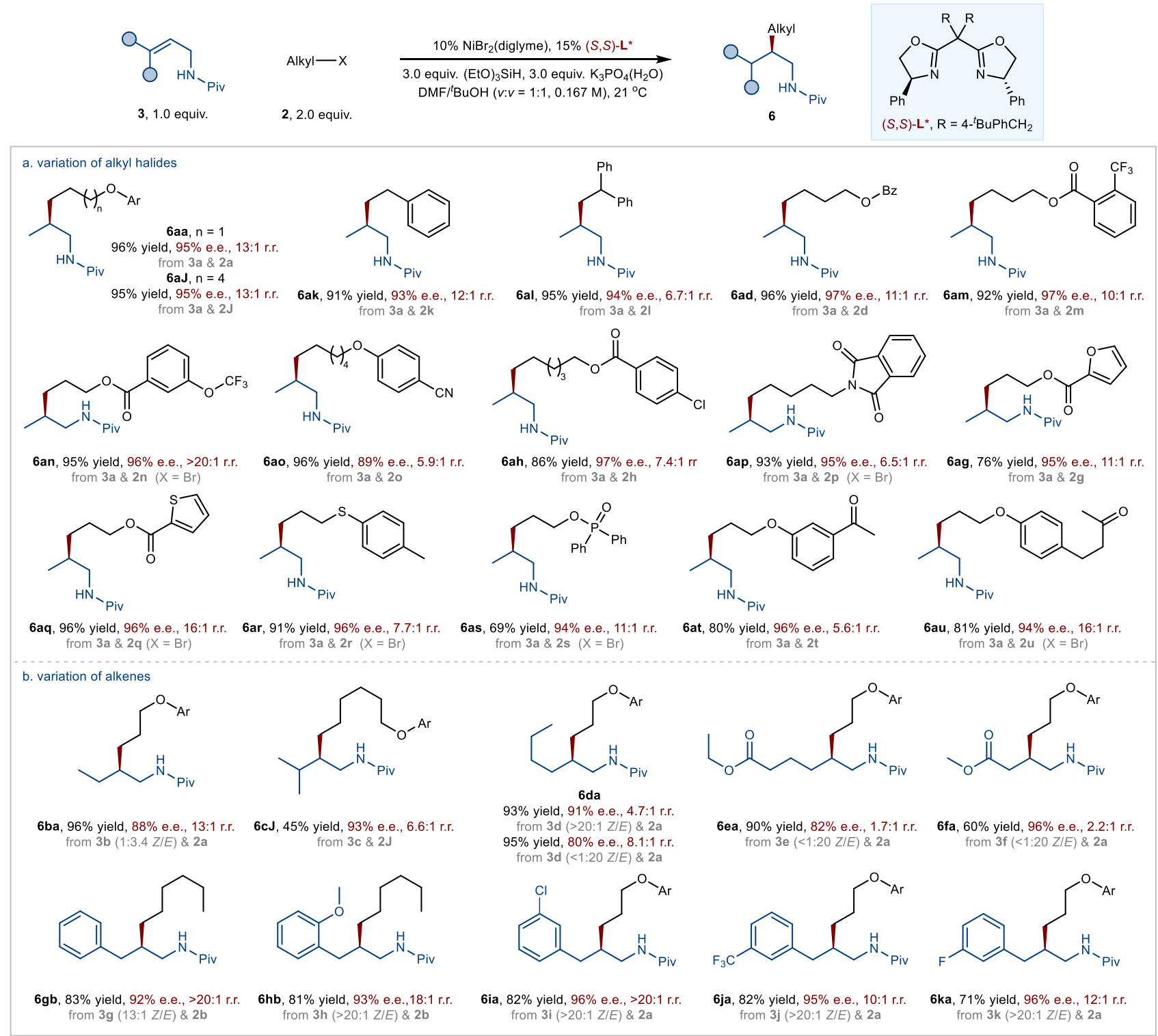

Figure 4. Enantioselective $\beta$-selective hydroalkylation. Method C: alkene (o.20 mmol, 1.o equiv), alkyl halide

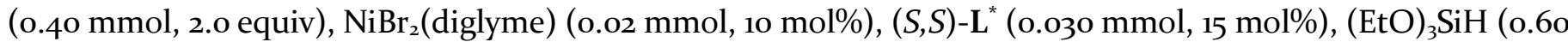
mmol, 3.o equiv), $\mathrm{K}_{3} \mathrm{PO}_{4}\left(\mathrm{H}_{2} \mathrm{O}\right)$ (o.6o mmol, 3.0 equiv), $\mathrm{DMF} /{ }^{t} \mathrm{BuOH}(v: v=1: 1,1.2 \mathrm{~mL}, 0.167 \mathrm{M}), 2{ }^{\circ} \mathrm{C}, 12 \mathrm{~h}$, isolated yield. The r.r. value was determined by GC or ${ }^{1} \mathrm{H}$ NMR analysis; the e.e. value was determined by HPLC analysis. 


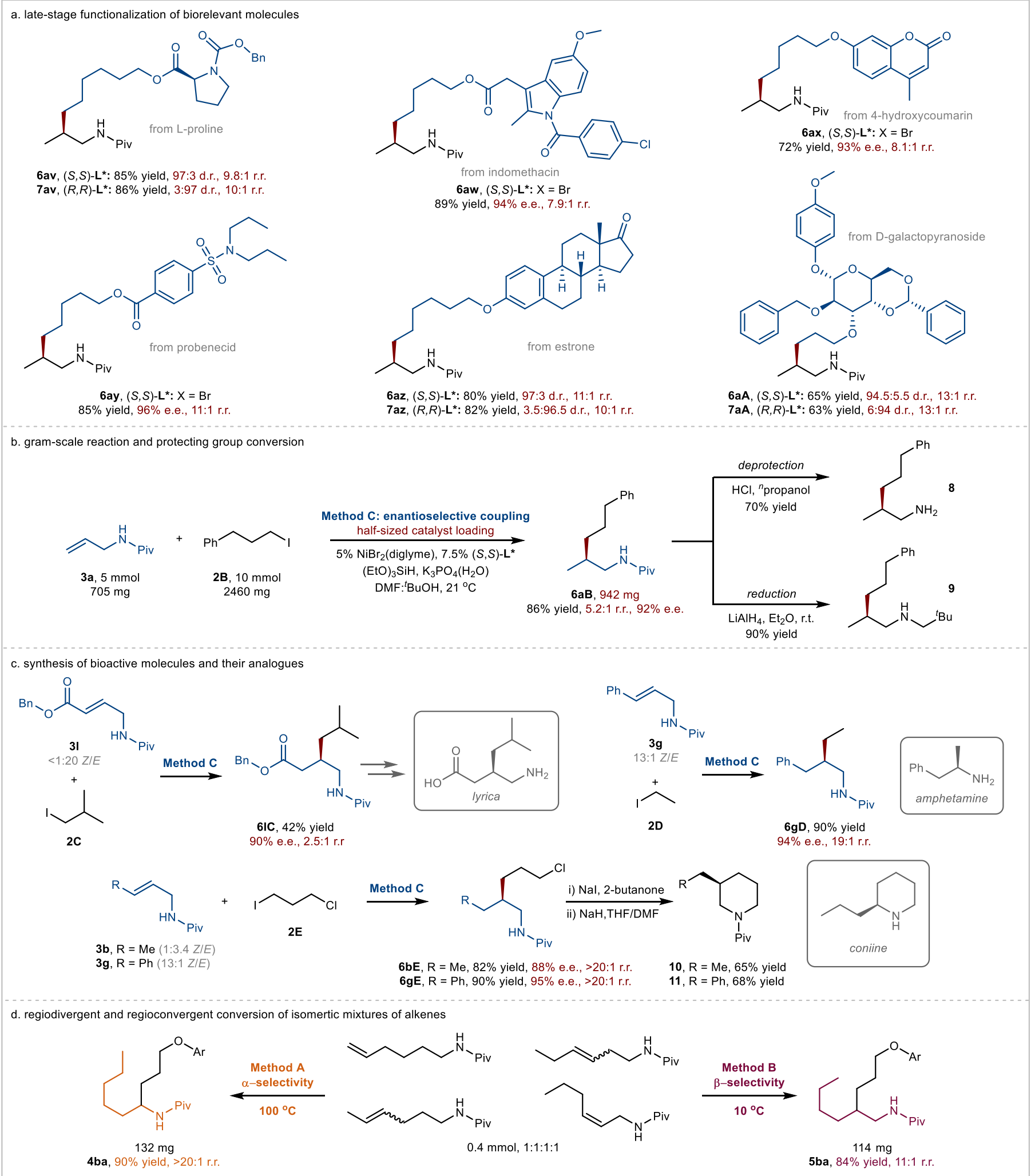

Figure 5. Synthetic applications. All yields refer to the total isolated yield for the mixture of all regioisomer purified products. The r.r. value was determined by GC or ${ }^{1} \mathrm{H}$ NMR analysis; the e.e. value was determined by HPLC analysis.

Synthetic applications. The enantioselective $\beta$-selective hydroalkylation exhibited good compatibility with many functional groups and was perfectly compatible for the late-stage functionalization of biorelevant 
molecules (Figure 5a). Derivatives of indomethacin (2w), coumarin (2x), and probenecid (2y) were smoothly reacted with alkene $3 \mathbf{a}$ to provide the desired products (6aw, 6ax, and 6ay) in good yield (72 89\%) and selectivity (93 96\% e.e. and 7.9:1 11:1 r.r.). In the modification of natural products L-proline (2v), estrone (2z), and D-galactopyranoside (2A), we obtained diastereoisomers (6av and 7av, $6 \mathbf{a z}$ and $7 \mathbf{a z}, \mathbf{6 a A}$ and $7 \mathbf{a A}$ ) using the proper enantiomer of the ligand. The preloaded chiral centres in the natural products hardly affect the coupling efficiency and regio- and enantioselectivity of the reaction. In terms of scalability, the synthesis of product $\mathbf{6 a B}$ could be conducted on a gram scale with an $86 \%$ yield (Figure 5 b). After hydrolysis of $\mathbf{6 a B}$ with $\mathrm{HCl}$ in propanol, deprotected primary alkyl amine 8 was obtained in a $70 \%$ isolated yield. Reduction of $\mathbf{6 a B ~ b y ~} \mathrm{LiAlH}_{4}$ afforded the corresponding secondary amine 9 in a 90\% isolated yield. The scale-up reaction and easy removal of the protecting group highlight the practicality of this $\beta$-branched alkyl amine synthesis. We applied this reaction to synthesize intermediates of bioactive molecules and their analogues (Figure 5c). Compound $6 \mathbf{C l C}$ was prepared as the intermediate of the acesodyne medication Lyrica, a $\gamma$-aminobutyric acid (GABA) receptor agonist. We also synthesized an elongated analogue (6gD) of amphetamine (a synthetic stimulant-type medication) and a structural analogue (10 and $\mathbf{1 1}$ ) of coniine (a polyketide-derived alkaloid) in short synthesis steps. We further demonstrated the utility of this temperature-regulated migratory hydroalkylation by employing isomeric mixtures of alkenes (Figure $5 \mathrm{~d}$ ). Our protocol exhibited both regiodivergence and regioconvergence, delivering each predictable regioselective product $\mathbf{4} \mathbf{b a}$ or $\mathbf{5} \mathbf{b a}$ with high regioselectivity from isomeric mixtures of the alkene substrates.

Mechanistic considerations. A set of radical clock experiments using (bromomethyl)cyclopropane (2F) or 6iodohex-1-ene $(\mathbf{2} \mathbf{G})$ was carried out (Figure $6 \mathrm{a}$ ). We observed the ring-opened product (6aF) from the cyclopropylmethyl radical and the cyclized products $(\mathbf{4 a G}, 6 \mathbf{a G})$ from the 5 -hexenyl radical. Furthermore, the ratio of uncyclized $\left(\mathbf{6} \mathbf{a} G^{\prime}\right) /$ cyclized $(\mathbf{6 a G})$ products exhibited a linear correlation with the nickel catalyst concentration. These observations indicated the noncage radical nature of this reaction.

A series of conventional deuterium labelling experiments further improved our understanding of the detailed reaction mechanism. The migratory hydroalkylation of the alkene ra was conducted under both method A and method B using $\mathrm{Ph}_{2} \mathrm{SiD}_{2}$ (Figure 6b). We observed the incorporation of deuterium at the $\gamma$ and $\delta$ positions of the amide group, and no H/D exchange was observed at the $\alpha$ or $\beta$ position of the amide group. The deuterium content at the $\delta$ position is always higher than that at the $\gamma$ position, indicating that Markovnikov- (rather than anti-Markovnikov-) hydrometallation is favoured. No $\alpha$ - or $\beta$-deuterium atom was observed, thus excluding the possibility of nickel walking back and forth along the alkyl chain. Furthermore, $\beta$-located alkyl-nickel could participate in cross-coupling or migrate to the $\alpha$ position but could not migrate to the outer $\gamma$-position. A $\delta, \delta$ dideuterated alkene $\left(\delta, \delta-d_{2}-\mathbf{r a}\right)$ was reacted with the alkyl halide $\mathbf{2 a}$ under method A and method B separately 
(Figure 6c). It is interesting to note that the deuterium content at the $\delta$-position decreased slightly, and no H/D exchange was observed along the chain. This phenomenon indicated that the $\gamma$-located nickel prefers chain walking toward the amide group to from $\alpha$ - or $\beta$ - located alkyl-nickel species. A $\beta, \beta$-dideuterated alkene $\left(\beta, \beta-d_{2^{-}}\right.$ 1a) was reacted with alkyl halides $2 \mathbf{k}$ and $2 \mathbf{H}$ (Figure $6 \mathrm{~d}$ ). The $\beta$ - and $\gamma$-position deuterated products $d_{2}-4$ ak and $d_{2}-5 \mathrm{aH}$ were obtained, respectively, and no deuteration was observed at the $\alpha$ position of the amide group. This conclusion was similar to that obtained from Figure 6c; nickel walking back and forth along the alkyl chain was less likely; in particular, $\alpha$-located alkyl-nickel could not migrate to the outer $\beta$ - or $\gamma$-position. We studied the stereochemistry of the asymmetric catalytic process using $\mathrm{Ph}_{2} \mathrm{SiD}_{2}$ to conduct deuterium-labelling experiments (Figure 6e). Under method C, 3a smoothly reacted with $\mathbf{2 k}$ to deliver the deuterated product $d_{1}$-6ak using $\mathrm{Ph}_{2} \mathrm{SiD}_{2}$. Deuterium was incorporated exclusively at the $\gamma$-position of the amide substituent. Thus, in this case, anti-Markovnikov-hydrometallation was completely forbidden. We observed a highly matched $Z / E$ ratio of the starting material alkene (3g) and diastereoselectivity of products (6gI and $\mathbf{6 g I}$ ') using either optically pure ligand or racemic ligand. We suggested that irreversible syn-hydrometalation was the regio- and enantio-determining step. In total, we could conclude that nickel chain walking was not in a back-and-forth modality but in a unidirectional modality, i.e., toward the amide group and to form the $\alpha$ - or $\beta$-located alkyl-nickel species. 


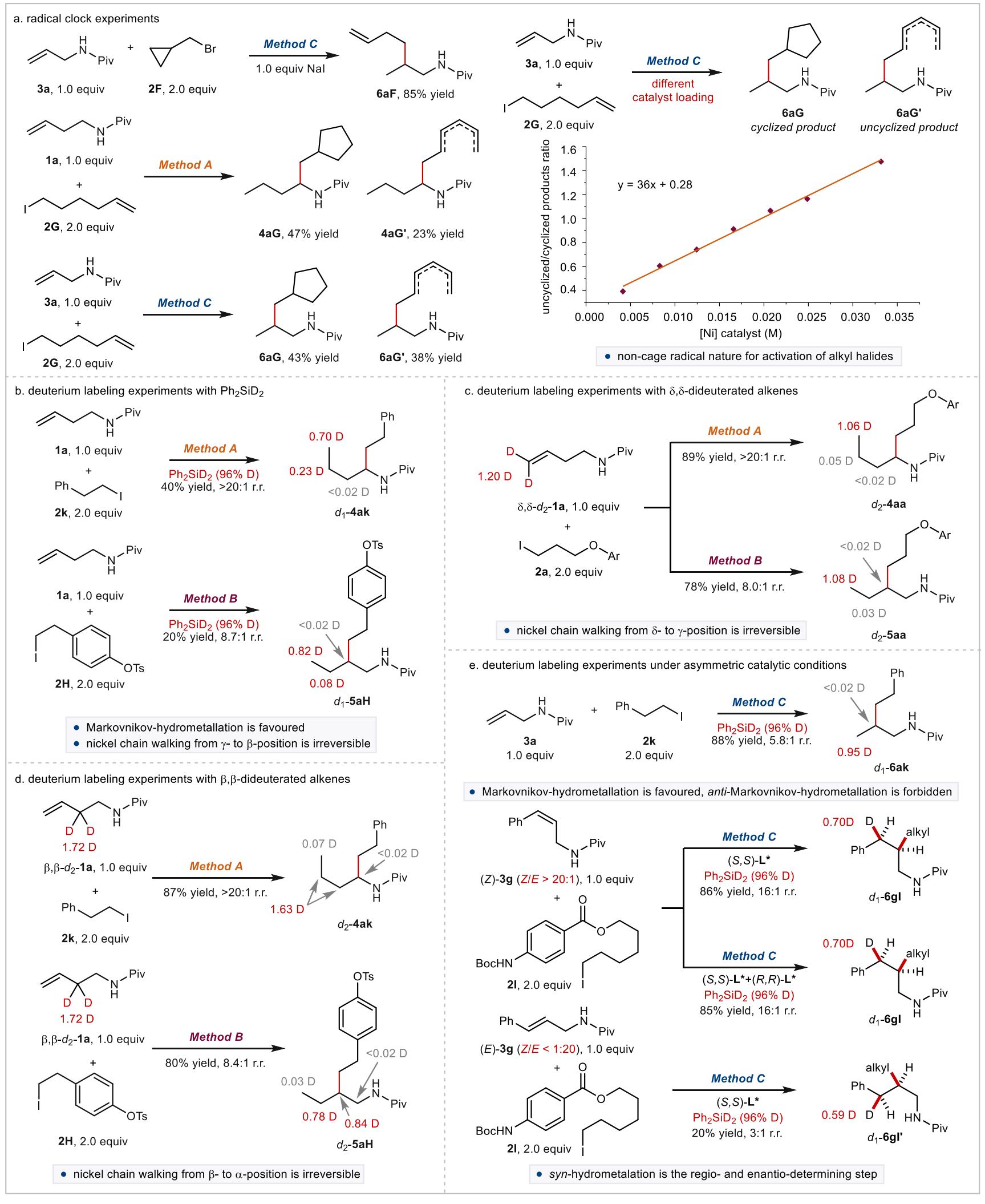

Figure 6. Radical clock experiments and deuterium labeling experiments. 


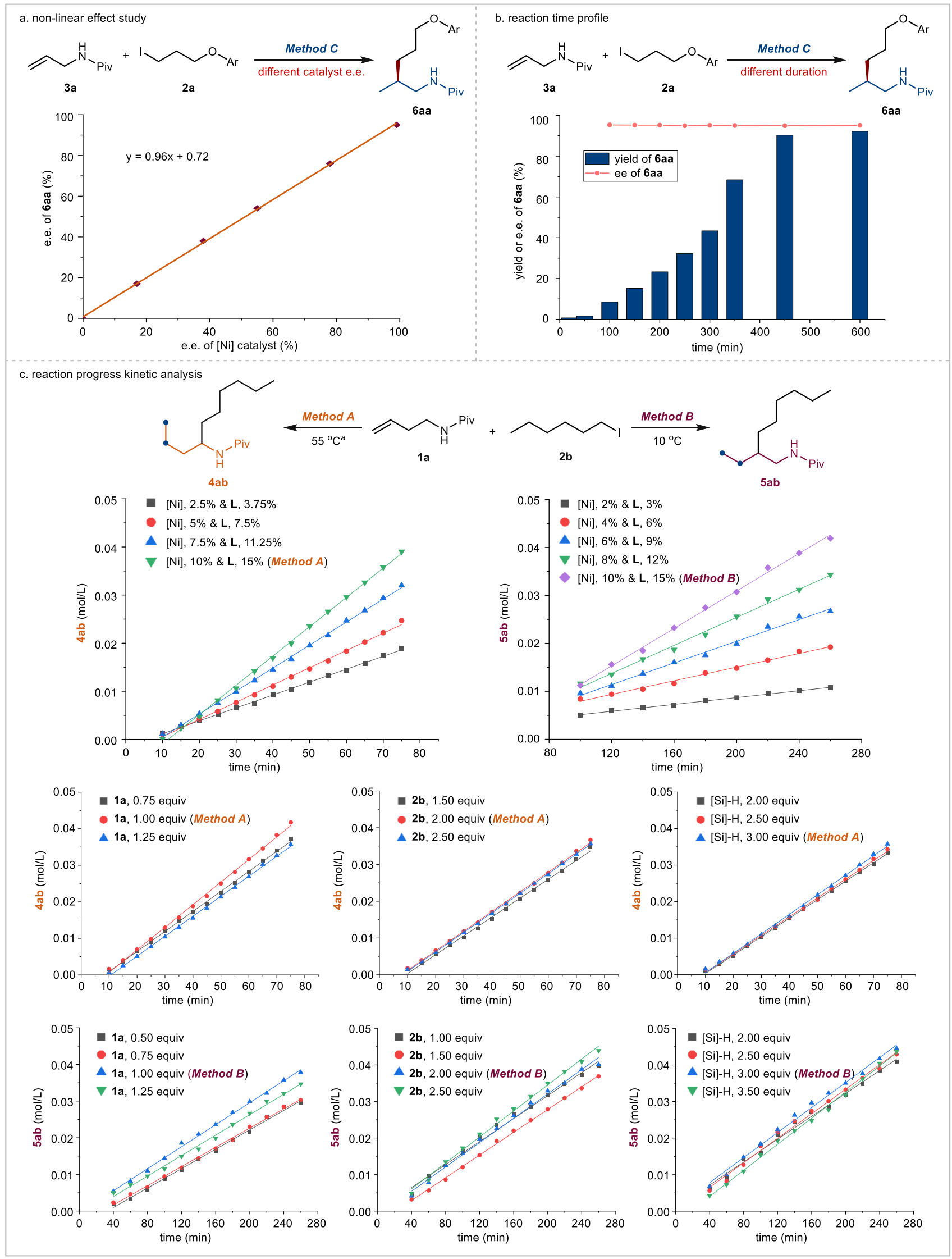

Figure 7. Catalyst characterization and reaction progress kinetic analysis. ${ }^{a}$ Reaction progress kinetic analysis for Method A was carried out at $55^{\circ} \mathrm{C}$ for a better balance between reasonable regioselectivity and reaction rate.

Catalyst characterization and reaction kinetics. More information about the asymmetric catalytic process 
was obtained through catalyst characterization and reaction progress kinetic analysis. The enantiopurities of the ligands used and the obtained hydroalkylation products exhibited a linear relationship (Figure 7a). Therefore, a monomeric nickel complex and a single bisoxazoline ligand were involved in alkene hydrometalation as the enantio-determining step. In addition, the enantiomeric excess of the desired product would not be expected to change during the reaction process (Figure $\mathrm{7b}$ ). Reaction progress kinetic analysis (RPKA) of the model reaction (Figure 7c, see Supplementary Figures 6-21 for more details) was conducted. Different initial concentrations of alkene $\mathbf{1 a}$, alkyl iodide $\mathbf{2} \mathbf{b}$ and hydrosilane $(\mathrm{EtO})_{3} \mathrm{SiH}$ could barely affect the reaction rate under both method $\mathrm{A}$ and method B conditions, indicating zero-order kinetics. We employed the Burés graphical rate analysis to compare the relationship of the reaction rate and catalyst concentration. The alkene migratory hydroalkylation has a rate order of 0.58 for nickel catalyst under method A conditions, while has a rate order of 1.05 for nickel catalyst under method B conditions (see Supplementary Figures $6 \& 14$ for more details). ${ }^{40}$ These observations indicated that both the oxidation addition of alkyl iodide and the hydrometallation were nonrate-determining steps. 


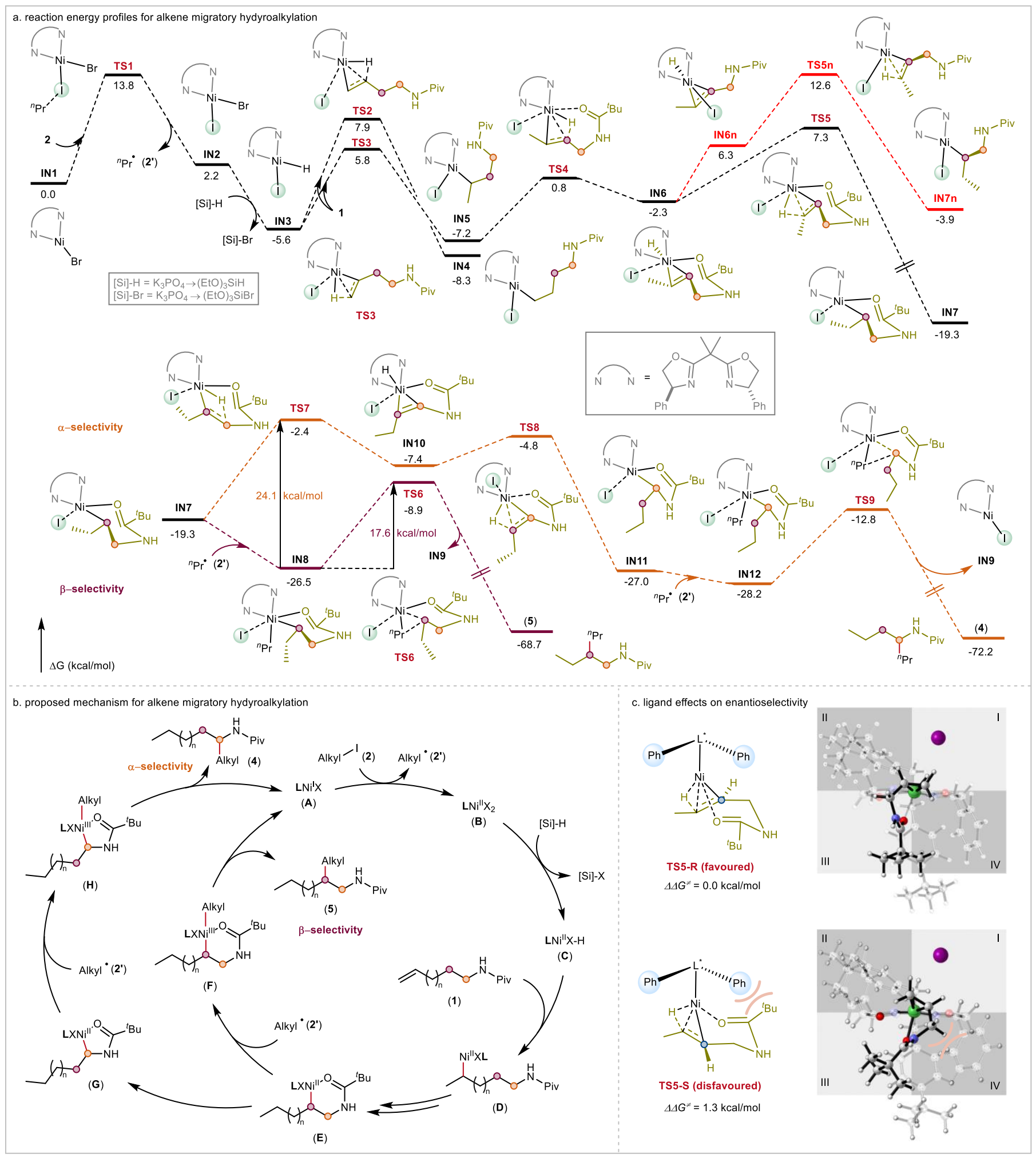

Figure 8. DFT calculations and the proposed mechanism. DFT calculations at the Mo6L-D3/6-311+G(d,p)-SDDSMD(DMA)//B3LYP-D3/6-31G(d)-SMD(DMA) level of theory. Free energies are given in kcal/mol.

DFT calculations. This alkene migratory hydroalkylation protocol exhibits temperature-regulated switchable site selectivity. It would be beneficial to elucidate the regio- and enantio-determining step for a profound understanding of the reaction mechanism and to illuminate more related temperature-regulated regiodivergent reactions. Density functional theory (DFT) calculations were conducted on a simplified reaction model. The 
computational results are shown in Figure 8a, and the catalytic cycle for the proposed mechanism is shown in Figure $8 \mathrm{~b}$. The $\mathrm{Ni}(\mathrm{I})$ complex (INı in Figure $8 \mathrm{a}, \mathbf{A}$ in Figure $8 \mathrm{~b}$ ) was suggested to be the initial catalyst under reductive conditions, generated by $\mathrm{Ni}(\mathrm{II}) \rightarrow \mathrm{Ni}(\mathrm{o})$ reductive elimination and the comproportionation of $\mathrm{Ni}(\mathrm{II})$ and $\mathrm{Ni}(\mathrm{o})$ species. ${ }^{15} \mathrm{Ni}(\mathrm{I}) \mathrm{X}(\mathrm{A})$ reacted with an alkyl halide (2) to afford $\mathrm{Ni}(\mathrm{II}) \mathrm{X}_{2}$ (IN2 in Figure 8a, B in Figure $8 \mathrm{~b})$ and the corresponding alkyl radical (2') through halogen-atom abstraction. $\mathrm{Ni}(\mathrm{II}) \mathrm{X}_{2}$ (B) reacted with hydrosilane to deliver a $\mathrm{Ni}(\mathrm{II}) \mathrm{H}$ complex (IN3 in Figure 8a, $\mathbf{C}$ in Figure $8 \mathrm{~b}$ ) under the promotion of $\mathrm{K}_{3} \mathrm{PO}_{4}$. Then, $\mathrm{Ni}(\mathrm{II}) \mathrm{H}(\mathbf{C})$ was inserted into the alkene (1) C-C double bond, resulting in nickel-alkyl species (IN 4 and IN $_{\mathbf{5}}$ in Figure 8a). The energy barrier difference between $\mathbf{T S}_{2}$ and $\mathbf{T S}_{3}\left(\Delta \Delta G^{\neq}=2.1 \mathrm{kcal} / \mathrm{mol}\right)$ indicated modest antiMarkovnikov-/Markovnikov- selectivity for $\beta$-migratory insertion and was consistent with the outcomes in Figure 6b. The Markovnikov-selective hydrometallation intermediate IN5 (D) was favoured due to the lower energy barrier of $\operatorname{TS}_{3}\left(\Delta G^{\ddagger}=11.4 \mathrm{kcal} / \mathrm{mol}\right)$. Rapid $\beta$-hydride elimination and inversed $\beta$-migratory insertion resulted in a relatively stable six-membered nickelacycle (IN7 in Figure 8a, E in Figure 8b). The energy demand for another proposed pathway involving a nondirected mechanism (IN6 $\rightarrow$ IN6 $\left.\mathbf{n} \rightarrow \mathbf{T S}_{\mathbf{5}} \mathbf{n} \rightarrow \mathbf{I N} \mathbf{7} \mathbf{n}\right)$ was also examined. The calculation results in the red line indicated that the energy barriers of the transition states in this proposed pathway were much higher than those in the directed mechanism $\left(\Delta \Delta G^{\ddagger}=5.3 \mathrm{kcal} / \mathrm{mol}\right)$. It became clear that the amide directing group was important for the lowering of the activation barrier for $\beta$-hydride elimination and inversed $\beta$-migratory insertion through coordination with the nickel centre. The sudden energy barrier reduction in $\mathbf{T S}_{5} \rightarrow \mathrm{IN}_{7}$ made it an irreversible step $(\Delta G=-26.6 \mathrm{kcal} / \mathrm{mol})$. This result was consistent with the outcomes in Figure $6 \mathrm{~b}$ that $\beta$-located alkyl-nickel could not migrate to the outer $\gamma$-position. The phenomenon observed in Figure $6 \mathrm{c}$ could be attributed to the much lower energy for $\mathrm{IN}_{7}$ (compared to that of $\mathbf{I N}_{4}$ or $\mathbf{I N}_{\mathbf{5}}$ ) that as the strong driving force of directional migration.

According to the computational results, the switchable site selectivity was generated in the subsequent transformation of $\mathbf{I N}_{7}$ (E in Figure $8 \mathrm{~b}$ ). Under a low reaction temperature, corresponding to migratory $\beta$ selective hydroalkylation, IN7 captures radical 2' to produce IN8 (F in Figure 8b). Subsequently, IN8 underwent reductive elimination to provide a $\beta$-selective hydroalkylation product (5) and regenerate the $\mathrm{Ni}(\mathrm{I}) \mathrm{X}$ catalyst (A) by surmounting a reaction energy barrier of $17.6 \mathrm{kcal} / \mathrm{mol}$. The rate-determining step for $\beta$-selective hydroalkylation was the reductive elimination step. For migratory $\alpha$-selective hydroalkylation, ring contraction from a six-membered nickelacycle (IN7, E) to a five-membered nickelacycle (IN11 in Figure 8a, G in Figure 8b) was necessary. The high energy barrier of $\mathbf{2 4 . 1} \mathrm{kcal} / \mathrm{mol}$ (more stable $\mathbf{I N 8} \rightarrow \mathbf{T S}_{\mathbf{7}}$, rather than $\mathbf{I N}_{\mathbf{7}} \rightarrow \mathbf{T S}_{\mathbf{7}}$ ) was in accordance with the $100{ }^{\circ} \mathrm{C}$ reaction temperature. The five-membered nickelacycle $\mathbf{I N 1 1}(\mathbf{G})$ captured the alkyl radical (2') to form a Ni(III) species (IN12 in Figure 8a, H in Figure 8b). The Ni(III) species (IN12, H) underwent reductive elimination to deliver the $\alpha$-selective hydroalkylation product (4) and regenerate the $\mathrm{Ni}(\mathrm{I}) \mathrm{X}$ catalyst 
(A). The driving force for migratory $\alpha$-selective hydroalkylation was the relatively lower (by $1.7 \mathrm{kcal} / \mathrm{mol}$ ) energy barrier for reductive elimination from IN12 compared to that of IN8 ( $\beta$-selective). The rate-determining step for $\alpha$-selective hydroalkylation was the $\beta$-hydride elimination step $\left(\mathbf{I N 8} \rightarrow \mathbf{T S}_{\mathbf{7}}\right)$. The sudden energy decrease of $\mathbf{T S}_{\mathbf{7}}$ $\rightarrow$ IN1o $\rightarrow$ TS8 $\rightarrow$ IN11 made it an irreversible step. This result was consistent with the outcomes in Figure $6 \mathrm{~d}$ that $\alpha$-located alkyl-nickel could not migrate to the outer $\beta$ - or $\gamma$-position. In total, the thermodynamic stability of IN12 over IN8 was responsible for the $\alpha$-selective hydroalkylation, while the kinetics advantage of IN8 $\rightarrow$ TS6 over IN8 $\rightarrow$ TS $_{7}$ was responsible for the $\beta$-selective hydroalkylation. In addition, the computational results reasonably explained our conclusion in Figure $6 \mathrm{~b} \sim \mathrm{d}$ that the chain walking of nickel was not in a back-and-forth modality but in a unidirectional modality, i.e., toward the amide group and to form the $\alpha$ - or $\beta$-located alkyl-nickel species. Notably, we also proposed a Ni(I)H-involved mechanistic variant. However, the high energy barrier $\left(\right.$ IN7 $_{7} \rightarrow$ TS $_{7}$, 31.o kcal/mol; see Supplementary Figure 25) for $\beta$-H elimination on a stable six-membered nickelacycle made this variant relatively less probable. In addition, DFT calculations were also carried out to explain the formation of the unexpected linear product $\mathbf{5} \mathbf{f a}$ at low reaction temperature (Supplementary Figure 26).

According to our deuterium labelling experiments shown in Figure 6e, the enantio-determining step was revealed as the hydrometallation step, i.e., the migratory insertion of $\mathrm{Ni}(\mathrm{II}) \mathrm{H}$ to produce $\mathrm{IN}_{7}(\mathbf{E})$. A comparison of TS5-S and TS5-R was conducted and is shown in Figure 8c. TS5-S was thermodynamically disfavoured, which could be attributed to the repulsion between the aryl substituent group in the ligand and the alkyl group in the amide protecting group. It can also be seen from the quadrant diagram obtained perpendicular to the Ni-ligand direction that the ligands are mainly distributed in quadrants II and IV. For TS-S, the alkyl chain has steric repulsion with the ligand in quadrant IV, making it relatively unstable. The feasibility of $\mathbf{T S}_{\mathbf{5}}-\mathbf{R}$ resulted in the major S-configuration products.

\section{Conclusions}

We disclose a temperature-regulated regiodivergent route for $\mathrm{C}\left(s p^{3}\right)-\mathrm{C}\left(s p^{3}\right)$ bond formation, a scarce phenomenon in transition-metal-catalysed organic synthesis. Regiodivergent alkylation was realized by varying only the reaction temperatures of alkene migratory hydroalkylation starting from the same raw materials, the identical alkene and alkyl halide substrates, using the same nickel-hydride catalytic system. This reaction also exhibits regioconvergence, delivering the predictable regioselective product with high regioselectivity from the arbitrary isomer or even isomeric mixtures of the alkene substrates. This protocol allows for the convenient synthesis of $\alpha$ - and $\beta$-branched alkyl amines, each of which are substantially important to the pharmaceutical chemistry and biochemistry fields. Detailed mechanistic studies revealed that, the formation of more stable nickelacycle provides the strong driving force of directional migration and the thermodynamic and kinetic 
properties of different reduction elimination intermediates are responsible for the switchable site-selectivity. Thermodynamic stability of the corresponding reduction elimination intermediate is responsible for the $\alpha$ selective hydroalkylation, and kinetics advantage is crucial for the $\beta$-selective hydroalkylation. This research work confirms the opportunity and possibility that reaction temperature, one of the most easily changed physical variables in organic synthesis, can serve as a determining parameter in alkene (migratory) functionalization. We believe that temperature-regulated regiodivergent mode might be generalizable in metal-catalysed alkene functionalization, providing quick and convenient synthetic routes to diversified molecules.

\section{Methods}

General procedure for temperature-regulated migratory hydroalkylation. In the air, a $10 \mathrm{~mL}$ screw-cap test tube equipped with a magnetic stirrer was charged with $\mathrm{NiBr}_{2}($ diglyme) ( $0.02 \mathrm{mmol}, 10 \mathrm{~mol} \%)$, $\mathrm{L}$ (o.03 mmol, $15 \mathrm{~mol} \%)$ and tri-potassium phosphate monohydrate ( $0.6 \mathrm{mmol}$, 3.o eq.). The test tube was evacuated and backfilled with argon for three times, then DMAc $(1.2 \mathrm{~mL})$ was added. After the reaction solution was cooled to $\mathrm{o}^{\circ} \mathrm{C},(\mathrm{EtO})_{3} \mathrm{SiH}$ (o.6 mmol, 3.o eq.) was added dropwise via syringe, then the reaction solution was kept stirring for $15 \mathrm{~min}$ at $40^{\circ} \mathrm{C}$ until the system color turns dark brown. Next, alkyl halide (o.4 mmol, 2.o eq.) and alkene (o.2 mmol, 1.o eq.) were added after the resulting solution was stirred for $2 \min$ at $\mathrm{o}{ }^{\circ} \mathrm{C}$, then the solution was stirred at $100{ }^{\circ} \mathrm{C}$ or $10{ }^{\circ} \mathrm{C}$ for $12 \mathrm{~h}$. The reaction mixture was diluted with $\mathrm{H}_{2} \mathrm{O}$ followed by extraction with EtOAc, dried with anhydrous $\mathrm{Na}_{2} \mathrm{SO}_{4}$ and concentrated in vacuo. The residue was purified by flash column chromatography on silica gel to give the target product.

General procedure for enantioselective $\beta$-selective hydroalkylation. In the air, a $10 \mathrm{~mL}$ screw-cap test tube equipped with a magnetic stirrer was charged with $\mathrm{NiBr}_{2}$ (diglyme) (o.o2 mmol, $\left.10 \mathrm{~mol} \%\right),(S, S)-\mathbf{L}^{*}$ (o.o3 mmol, $15 \mathrm{~mol} \%)$ and tri-potassium phosphate monohydrate ( $.6 \mathrm{mmol}$, 3.o eq.). The test tube was evacuated and backfilled with argon for three times, then solvent (DMF: $\left.{ }^{\mathrm{BuOH}}=1: 1,1.2 \mathrm{~mL}\right)$ was added. After the reaction solution was cooled to $\mathrm{o}^{\circ} \mathrm{C}$, alkyl halide (o.4 mmol, 2.0 eq.) and alkene ( $0.2 \mathrm{mmol}$, 1.o eq.) were added. Next, $(\mathrm{EtO})_{3} \mathrm{SiH}$ (o.6 mmol, 3.o eq.) was added dropwise via syringe, the reaction solution was kept stirring for $5 \mathrm{~min}$ at $\mathrm{o}{ }^{\circ} \mathrm{C}$, then the solution was stirred at $21{ }^{\circ} \mathrm{C}$ for $12 \mathrm{~h}$. The reaction mixture was diluted with $\mathrm{H}_{2} \mathrm{O}$ followed by extraction with EtOAc, dried with anhydrous $\mathrm{Na}_{2} \mathrm{SO}_{4}$ and concentrated in vacuo. The residue was purified by flash column chromatography on silica gel to give the target product.

\section{Data availability}

The authors declare that the data supporting the findings of this study are available within the article and Supplementary Information files or from the corresponding author upon reasonable request. The experimental 
procedures, computational results and characterization of all new compounds are provided in the Supplementary Information.

\section{Acknowledgements}

Financial support from the National Natural Science Foundation of China (grant numbers 21732006 and 21927814), the USTC Research Funds of the Double First-Class Initiative (grant number YD3530002002) and the CAS Collaborative Innovation Program of Hefei Science Center (grant number 2021HSC-CIPoo4).

\section{Author contributions}

X.L. directed the project, conceived the idea and wrote the manuscript. J.-W.W., Z.C. and Z.L. performed the synthetic experiments. Y.F. directed and D.-G.L. performed the DFT calculations. All of the authors participated in the discussion and preparation of the manuscript.

\section{Competing interests}

The authors declare no competing interests.

\section{References}

(1) Nájera, C.; Beletskaya, I. P.; Yus, M. Metal-catalyzed regiodivergent organic reactions. Chem. Soc. Rev. 2019, 48, 4515-4618.

(2) Sakakibara, Y.; Murakami, K. Switchable Divergent Synthesis Using Photocatalysis. ACS Catal. 2022, $1857-1878$.

(3) Juliá-Hernández, F.; Moragas, T.; Cornella, J.; Martin, R. Remote carboxylation of halogenated aliphatic hydrocarbons with carbon dioxide. Nature 2017, 545, 84-88.

(4) Lovering, F. Escape from Flatland 2: complexity and promiscuity. Med. Chem. Commun. 2013, 4, 515-519.

(5) Geist, E.; Kirschning, A.; Schmidt, T. $s p^{3}-s p^{3}$ Coupling reactions in the synthesis of natural products and biologically active molecules. Nat. Prod. Rep. 2014, 31, 441-448.

(6) Boström, J.; Brown, D. G.; Young, R. J.; Keserü, G. M. Expanding the medicinal chemistry synthetic toolbox. Nat. Rev. Drug Discov. 2018, 17, 709-727.

(7) Pirnot, M. T.; Wang, Y.-M.; Buchwald, S. L. Copper Hydride Catalyzed Hydroamination of Alkenes and Alkynes. Angew. Chem. Int. Ed. 2016, 55, 48-57.

(8) Crossley, S. W.; Obradors, C.; Martinez, R. M.; Shenvi, R. A. Mn-, Fe-, and Co-Catalyzed Radical Hydrofunctionalizations of Olefins. Chem. Rev. 2016, 116, 8912-900o.

(9) Jordan, A. J.; Lalic, G.; Sadighi, J. P. Coinage Metal Hydrides: Synthesis, Characterization, and Reactivity. Chem. Rev. 2016, 116, 8318-8372.

(10) Wang, X.-X.; Lu, X.; Li, Y.; Wang, J.-W.; Fu, Y. Recent Advances in Nickel-Catalyzed Reductive Hydroalkylation and Hydroarylation of Electronically Unbiased Alkenes. Sci China Chem 2020, 63, 1586-160o.

(11) He, Y.; Chen, J.; Jiang, X.; Zhu, S. Enantioselective NiH-Catalyzed Reductive Hydrofunctionalization of Alkenes. Chin. J. Chem. 2022, 40, 651-661.

(12) Liu, R. Y.; Buchwald, S. L. CuH-Catalyzed Olefin Functionalization: From Hydroamination to Carbonyl Addition. Acc. Chem. Res. 2020, 53, 1229-1243.

(13) Maksymowicz, R. M.; Roth, P. M. C.; Fletcher, S. P. Catalytic asymmetric carbon-carbon bond formation 
using alkenes as alkylmetal equivalents. Nat. Chem. 2012, 4, 649-654.

(14) Lu, X.; Xiao, B.; Zhang, Z.; Gong, T.; Su, W.; Yi, J.; Fu, Y.; Liu, L. Practical carbon-carbon bond formation from olefins through nickel-catalyzed reductive olefin hydrocarbonation. Nat. Commun. 2016, 7, 11129.

(15) Wang, Z.; Yin, H.; Fu, G. C. Catalytic enantioconvergent coupling of secondary and tertiary electrophiles with olefins. Nature 2018, 563, 379-383.

(16) Li, Y.; Nie, W.; Chang, Z.; Wang, J.-W.; Lu, X.; Fu, Y. Cobalt-catalysed enantioselective C(sp3)-C(sp3) coupling. Nat. Catal. 2021, 4, 901-911.

(17) Bera, S.; Mao, R.; Hu, X. Enantioselective $\mathrm{C}(\mathrm{sp} 3)-\mathrm{C}\left(\mathrm{sp}_{3}\right)$ cross-coupling of non-activated alkyl electrophiles via nickel hydride catalysis. Nat. Chem. 2021, 13, 270-277.

(18) Massad, I.; Suresh, R.; Segura, L.; Marek, I. Stereoselective synthesis through remote functionalization. Nat. Synth. 2022, 1, 37-48.

(19) Vasseur, A.; Bruffaerts, J.; Marek, I. Remote functionalization through alkene isomerization. Nat. Chem. 2016, 8, 209-219.

(20) Sommer, H.; Juliá-Hernández, F.; Martin, R.; Marek, I. Walking Metals for Remote Functionalization. ACS Cent. Sci. 2018, 4, 153-165.

(21) Janssen-Müller, D.; Sahoo, B.; Sun, S.-Z.; Martin, R. Tackling Remote $s p^{3}$ C-H Functionalization via NiCatalyzed "chain-walking” Reactions. Isr. J. Chem. 2020, 6o, 195-206.

(22) Fiorito, D.; Scaringi, S.; Mazet, C. Transition metal-catalyzed alkene isomerization as an enabling technology in tandem, sequential and domino processes. Chem. Soc. Rev. 2021, 50, 1391-1406.

(23) Ghosh, S.; Patel, S.; Chatterjee, I. Chain-walking reactions of transition metals for remote C-H bond functionalization of olefinic substrates. Chem. Commun. 2021, 57, 11110-11130.

(24) Bair, J. S.; Schramm, Y.; Sergeev, A. G.; Clot, E.; Eisenstein, O.; Hartwig, J. F. Linear-Selective Hydroarylation of Unactivated Terminal and Internal Olefins with Trifluoromethyl-Substituted Arenes. J. Am. Chem. Soc. 2014, 136, 13098-13101.

(25) He, Y.; Cai, Y.; Zhu, S. Mild and Regioselective Benzylic C-H Functionalization: Ni-Catalyzed Reductive Arylation of Remote and Proximal Olefins. J. Am. Chem. Soc. 2017, 139, 1061-1064.

(26) Qian, D.; Hu, X. Ligand-Controlled Regiodivergent Hydroalkylation of Pyrrolines. Angew. Chem. Int. Ed. 2019, 58, 18519-18523.

(27) Zhang, Y.; He, J.; Song, P.; Wang, Y.; Zhu, S. Ligand-Enabled NiH-Catalyzed Migratory Hydroamination: Chain Walking as a Strategy for Regiodivergent/Regioconvergent Remote $s p^{3} \mathrm{C}-\mathrm{H}$ Amination. CCS Chem. 2020, 2, 2259-2268.

(28) Du, X.; Zhang, Y.; Peng, D.; Huang, Z. Base-Metal-Catalyzed Regiodivergent Alkene Hydrosilylations. Angew. Chem. Int. Ed. 2016, 55, 6671-6675.

(29) Miller, Z. D.; Dorel, R.; Montgomery, J. Regiodivergent and Stereoselective Hydrosilylation of 1,3Disubstituted Allenes. Angew. Chem. Int. Ed. 2015, 54, 9088-9091.

(30) Tani, Y.; Fujihara, T.; Terao, J.; Tsuji, Y. Copper-Catalyzed Regiodivergent Silacarboxylation of Allenes with Carbon Dioxide and a Silylborane. J. Am. Chem. Soc. 2014, 136, 17706-17709.

(31) Su, W.; Gong, T.-J.; Lu, X.; Xu, M.-Y.; Yu, C.-G.; Xu, Z.-Y.; Yu, H.-Z.; Xiao, B.; Fu, Y. Ligand-Controlled Regiodivergent Copper-Catalyzed Alkylboration of Alkenes. Angew. Chem. Int. Ed. 2015, 54, 12957-12961.

(32) Lei, H.; Conway, J. H.; Cook, C. C.; Rovis, T. Ligand Controlled Ir-Catalyzed Regiodivergent Oxyamination of Unactivated Alkenes. J. Am. Chem. Soc. 2019, 141, 11864-11869.

(33) Sakae, R.; Hirano, K.; Miura, M. Ligand-Controlled Regiodivergent Cu-Catalyzed Aminoboration of Unactivated Terminal Alkenes. J. Am. Chem. Soc. 2015, 137, 646o-6463.

(34) Dorn, S. K.; Brown, M. K. Cooperative Pd/Cu Catalysis for Alkene Arylboration: Opportunities for Divergent Reactivity. ACS Catal. 2022, 12, 2058-2063.

(35) Bai, X.-Y.; Wang, Z.-X.; Li, B.-J. Iridium-Catalyzed Enantioselective Hydroalkynylation of Enamides for the Synthesis of Homopropargyl Amides. Angew. Chem. Int. Ed. 2016, 55, 9007-9011.

(36) Choi, J.; Fu, G. C. Transition metal-catalyzed alkyl-alkyl bond formation: Another dimension in cross- 
coupling chemistry. Science 2017, 356, eaaf7230.

(37) He, S.-J.; Wang, J.-W.; Li, Y.; Xu, Z.-Y.; Wang, X.-X.; Lu, X.; Fu, Y. Nickel-Catalyzed Enantioconvergent Reductive Hydroalkylation of Olefins with $\alpha$-Heteroatom Phosphorus or Sulfur Alkyl Electrophiles. J. Am. Chem. Soc. 2020, 142, 214-221.

(38) Wang, J.-W.; Li, Y.; Nie, W.; Chang, Z.; Yu, Z.-A.; Zhao, Y.-F.; Lu, X.; Fu, Y. Catalytic asymmetric reductive hydroalkylation of enamides and enecarbamates to chiral aliphatic amines. Nat. Commun. 2021, 12, 1313.

(39) Wang, X.-X.; Yu, L.; Lu, X.; Zhang, Z.-L.; Liu, D.-G.; Tian, C.; Fu, Y. NiH-Catalyzed Reductive Hydrocarbonation of Enol Esters and Ethers. CCS Chem. 2021, 3, 727-737.

(40) Burés, J. A Simple Graphical Method to Determine the Order in Catalyst. Angew. Chem. Int. Ed. 2016, 55, 2028-2031. 